\title{
Optimal Allocation of Mould Manufacturing Resources Under Manufacturing Network Environments based on a Bi-Level Programming Model
}

\author{
Hongyan $\mathrm{Hao}^{\mathrm{a}, \mathrm{b}, *}$, Fanxin Kong ${ }^{\mathrm{a}, \mathrm{b}}$ \\ a Jiangsu Key Laboratory of Advanced Structural Materials and Application Technology, Nanjing, 211167, China \\ ${ }^{b}$ School of Material Engineering, Nanjing Institute of Technology, Nanjing, 211167,China
}

\begin{abstract}
In order to achieve the optimal selection and allocation of various mould manufacturing resources under a manufacturing network environment, this study developed a bi-level programming model and proposed a related solution. Based on a thorough analysis of the allocation process of mould manufacturing resources, the calculation formulas of the task's satisfaction with resource service quality and the load balance rate of resources were derived, and a bi-level programming mathematical model for the optimal allocation of mould manufacturing resources was established. Moreover, a hierarchical discrete particle swarm optimization (HDPSO) algorithm, which was based on the principle of non-dominated sorting in a multi-objective optimization, was designed for solving the model. Finally, a mould manufacturing project was selected for experimentally validating the feasibility of the proposed bi-level programming model for the optimal allocation of resources and the effectiveness of the proposed HDPSO algorithm.
\end{abstract}

Keywords: manufacturing network; bi-level programming; HDPSO algorithm; optimal allocation of resources

(Submitted on August 29, 2017; Revised on September 30, 2017; Accepted on October 18, 2017)

(C) 2017 Totem Publisher, Inc. All rights reserved.

\section{Introduction}

Due to the current globalization of the manufacturing industry, mould enterprises, which are classic examples of resourceoriented and order-driven organizations, should be able to respond quickly to market demands and complete customer orders promptly. However, most of the mould enterprises in China are small and medium-sized enterprises, and do not possess the various resources for manufacturing all types of mould. This means that many enterprises need to break down barriers, share resources and achieve the dynamic optimization and integration of manufacturing resources. Accordingly, not only would orders be fulfilled on schedule, but also resources could be utilized fully, so as to maximize economic benefits and increase profits. A manufacturing network, which is a new networked production model, was developed for precisely these reasons, and had the aim of pooling idle and superior manufacturing resources, which were originally scattered in different geographical locations, thereby achieving the effective utilization of resources. Therefore, gaining an in-depth knowledge of the optimal allocation of manufacturing resources under a manufacturing network environment is of great significance with regard to the implementation and development of a manufacturing network.

To date, a great amount of research has been conducted regarding the optimal allocation of manufacturing resources. For example, Fu Jingzhi et al. adopted some performance parameters including time (T), quality (Q), cost (C) and service (S) as the important evaluation indexes of the allocation of, and search for, manufacturing resources in a network environment, and then investigated the optimal manufacturing resources that satisfied the requirements of manufacturing tasks [1-5]. Furthermore, Zhou Changchun et al. also selected T, Q, C and S as the evaluation indexes that satisfied users' resource requirements, and made a feasible selection and optimal allocation of manufacturing resources using a two-stage fuzzy comprehensive evaluation [18]. Zhang Xiangbin et al. aimed at the minimization of the total cost of task completion

\footnotetext{
* Corresponding author.

E-mail address: haohongyan@njit.edu.cn.
} 
and established the optimal allocation model of resources [19]. Liu Lilan et al. used indexes of the resources' quality of service (QoS) attributes, namely, time $(\mathrm{T})$, price $(\mathrm{P})$, quality $(\mathrm{Q})$ and service $(\mathrm{S})$, as the evaluation criterion of resources, and proposed a resource scheduling method based on the QoS's group decision-making fuzzy-analytic hierarchy model [7, 8]. Ma Xuefen et al. established an optimal allocation model of manufacturing resources, in which product delivery time, cost and manufacturing quality were employed as three objectives, and then solved this model using a genetic algorithm (GA) [11]. Song Shuqiang et al. used cost and time as the optimization objectives of manufacturing resources and introduced a quantum-behaved particle swarm optimization (QPSO) algorithm for the solution [13]. Based on the analytic hierarchy process (AHP), Shi Zhanbei et al. constructed a network node selection method, which used T, Q, C and S as the optimization criterion of manufacturing resources, and attempted to select some optimal resource nodes [14]. Fei Tao et al. proposed a manufacturing grid resource service QoS model (including time, cost, reliability, and satisfaction) and QoSbased resource search method [15-17].

To summarize, previous studies mainly established evaluation models of manufacturing resources that only included time, quality, cost and service, which were related to manufacturing tasks, and then adopted several algorithms, which mainly included the PSO [8,11,13], GA [2, 4, 11], AHP [7,14] and two-stage fuzzy comprehensive evaluation [18], to solve the models. However, the load balance of mould manufacturing resources was not taken into account in these studies, which accordingly always yielded a local optimization scheme as opposed to a systematical optimization scheme that considered the manufacturing task and resources. Therefore, this study first reviewed research results from all over the world, and explored the feasibility of using a bi-level programming model to solve the optimal allocation problem of mould manufacturing resources in a network environment. Then, using the degree to which the task's requirements were satisfied (the task's satisfaction degree) with regard to resource service quality and the load balance of the manufacturing enterprise's resources as the upper-level and lower-level programming objectives respectively, a bi-level programming model for the optimal allocation of manufacturing resources was established, and an improved PSO algorithm embedding the bi-level iteration rule was proposed as a solution.

\section{Analysis of the Allocation Process of Mould Manufacturing Resources}

This study assumed that a mould manufacturing project existed in a manufacturing network environment, which included multiple manufacturing tasks. For each manufacturing task, many different manufacturing service resources can satisfy the task requirements. A lot of information, including delivery date, production cost and quality requirements, was first released for the manufacturing task via the network nodes. Then, the related assessment and screening activities took place under the constraint conditions of various criteria $(\mathrm{T}, \mathrm{C}, \mathrm{Q}$ and $\mathrm{S}$ ) in accordance with the feedback results of the resource manager; next, the candidate resource sets of various manufacturing tasks were acquired, and the information including delivery date, manufacturing cost and completion quality that was promised from the enterprise responsible for the manufacturing task were ascertained; finally, the optimization model of manufacturing resource selection was established so that each manufacturing task was assigned to the most appropriate manufacturing resource. Accordingly, the optimal allocation scheme of manufacturing resources that achieved the load balance of manufacturing resources and simultaneously maximized the task satisfaction degree of resource service quality was obtained. As shown in Fig. 1, a problem should be firstly addressed in the optimal allocation of resources, i.e., how to select the most appropriate service resource set from multiple candidate service resource sets for each task.

\section{The Bi-level Programming Model of the Optimal Allocation of Mould Manufacturing Resources}

\subsection{A mathematical description}

It is assumed that the manufacturing process of a set of moulds under a manufacturing network environment can be decomposed into $\mathrm{m}$ manufacturing tasks, denoted as $t=\left\{t_{1}, t_{2}, \cdots, t_{m}\right\}$, and these manufacturing tasks include many subtasks (for the i-th manufacturing task, its subtask set can be denoted as $t_{i}=\left\{t_{i 1}, t_{i 2}, \cdots, t_{i u_{i}}\right\}$ ); the set of candidate manufacturing resource nodes can be denoted as $G=\left\{G_{1}, G_{2}, \cdots, G_{n}\right\}$. The subtasks, with a total number of $M=\sum_{i=1}^{m} u_{i}$, were completed by n candidate resource nodes, in order to achieve the task's satisfaction degree with regard to resource service quality and the load balance of manufacturing resources.

\subsection{Hypotheses}

Hypothesis 1 - All candidate resource nodes that participate in mould manufacturing are equal. 
Hypothesis 2 - According to the principle that a single task cannot be decomposed, a task can only be processed at a resource service node.

Hypothesis 3 - The time of a candidate resource node required for fulfilling a manufacturing task includes the actual processing time and the logistics time for the transportation to the resource node.

Hypothesis 4 - The cost of a candidate manufacturing resource node required for fulfilling a manufacturing task includes the production cost and logistics cost.

Hypothesis 5 - The manufacturing network service cannot be interrupted. Once a manufacturing task is initialized, it cannot be stopped; in other words, the manufacturing service ability provided by the service nodes in the manufacturing network is continuous.

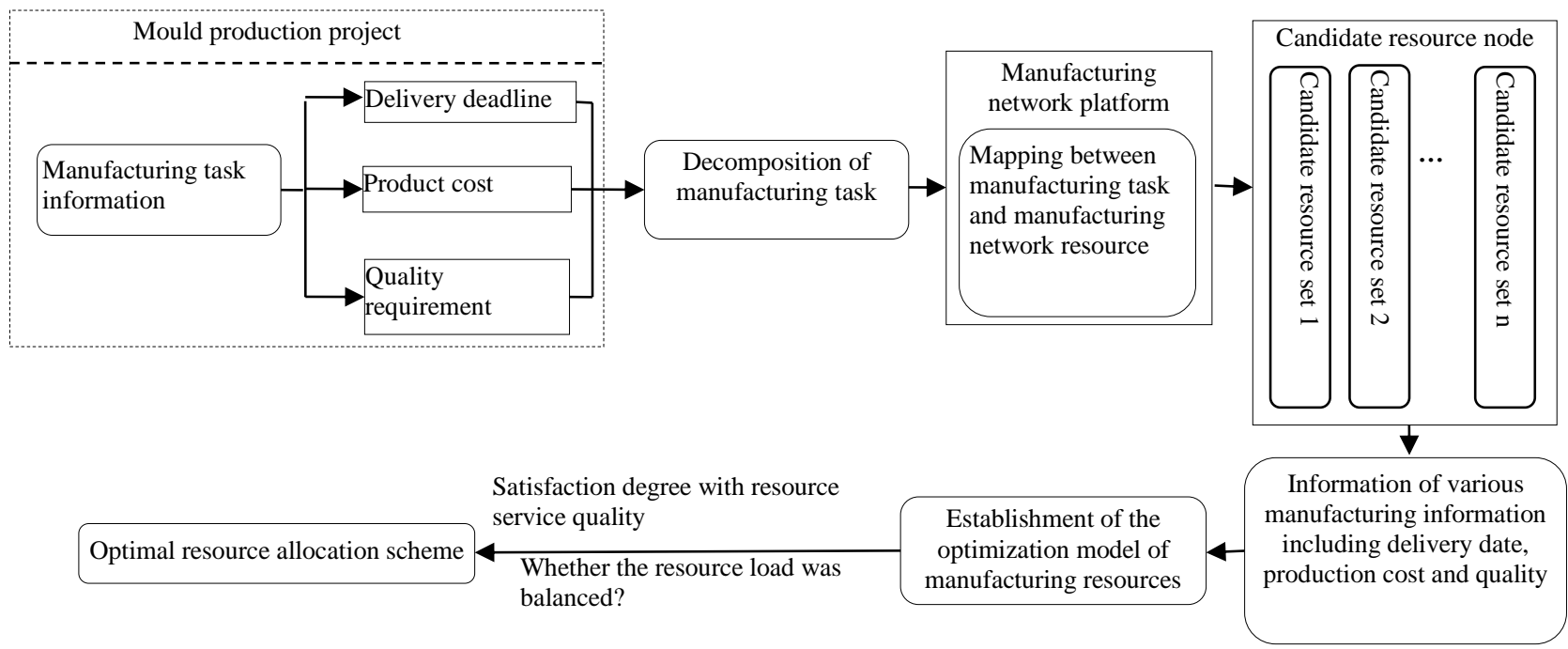

Figure 1. The optimal allocation process of manufacturing resources

\subsection{Definitions of the task's satisfaction degree with resource service quality and the load balance of manufacturing resources and the related calculation formulas}

When optimizing the allocation of manufacturing resources under a manufacturing network environment, the enterprises offering these resources should maximize the manufacturing task's satisfaction degree with regard to resource service quality, and simultaneously maintain the load balance of these resources as much as possible. However, maintaining the load balance of the enterprise's manufacturing resources would affect that task's satisfaction degree with resource service quality, while the task's satisfaction degree with resource service quality would also affect the load balance of the resources provided by manufacturing enterprises. Therefore, these two factors show mutual interdependence and interaction. Accordingly, the optimal allocation of manufacturing resources can be regarded as a hierarchical programming problem, which is generally described and solved using a bi-level programming model. Bi-level programming includes two levels of objectives and is a hierarchical optimization problem. The upper-level objective serves as the dominator while the lowerlevel objective serves as the subordinate. The objective function and constraint conditions in the upper level are not only related to the variables in this level, but are also affected by the optimal solution or optimal value in the lower-level programming; furthermore, the optimal solution in the lower-level programming is also subjected to the effects of the decisions made in the upper-level programming. In a decision-making process related to the optimal allocation of manufacturing resources, the satisfaction range of a manufacturing task with resource service quality should first be determined, and the acceptable range of various factors that affect the degree of satisfaction should be listed. Moreover, the enterprises offering manufacturing resources should seek the optimal scheme for the allocation of these resources.

\section{Definition 1: A task's satisfaction degree with resource service quality}

A task's satisfaction degree with resource service quality is also referred to as a task's satisfaction index with resource service quality, which is reflected by the comparison value between the actual feeling of the task's recipient in accepting resource services and their expectation, i.e., the difference between the perception quality and cognition quality. There are a 
number of evaluation methods regarding the task's satisfaction degree with resource service quality; however, the index measurement method was employed in this study for determining the key factors that affected the task's satisfaction degree with resource service quality. Subsequently, the weights of various factors $\left(C>C_{H}, C<C_{L}\right)$ were obtained, and the task's satisfaction index with resource service quality was calculated through the weighted summation:

$$
C S I=\sum_{j=1}^{p} W_{j} X_{j}
$$

where $\mathrm{p}$ denotes the number of key factors that affect the task's satisfaction degree with resource service quality, $W_{j}$ denotes the weight of the $j$-th factor and $X_{j}$ denotes the score of the $j$-th factor. In this study, only the influence factors of the customizing task's satisfaction degree with resource service quality, namely, delivery date (T), implementation cost (C) and completion quality (Q), were considered, and their weights were determined based on experience. Accordingly, CSI can be expressed as a function of T, C and Q:

$$
C S I=f(T, C, Q)
$$

Next, we focus on the relationships between the task's satisfaction degree with resource service quality and delivery date, implementation cost and completion quality.

(1) The relationship between the task's satisfaction degree with resource service quality and delivery date

For a task, the allowable delivery date is a certain time slot rather than a fixed time point. Therefore, the closer the actual delivery date is to the earliest delivery deadline, the higher the task's satisfaction degree. An advance and delayed delivery is not allowed; in these two cases, the task's satisfaction degree is equal to 0 . Within the task's allowable delivery date, the enterprises that offer manufacturing resources should deliver as early as possible, as the task's satisfaction degree with resource service quality will be greater; otherwise, the later the delivery, the lower the task's satisfaction degree with resource service quality. It was assumed that the task's satisfaction degree was the greatest and equal to 1 when the products were delivered on the earliest allowable delivery date, and the task's satisfaction degree was the lowest and equal to 0 when the products were delivered on the latest allowable delivery date. The following piecewise decreasing function was selected for describing the relationship between the task's satisfaction degree with resource service quality and delivery date:

$$
f_{1}\left(T_{i}\right)=\left\{\begin{array}{lr}
0 & T_{i}>T_{i H}, T_{i}<T_{i L} \quad i=1,2, \cdots, m \\
\frac{T_{i H}-T_{i}}{T_{i H}-T_{i L}} & T_{i L}<T_{i}<T_{i H}
\end{array}\right.
$$

where $T_{i H}$ and $T_{i L}$ denote the specified earliest and latest delivery dates respectively, i.e., the task's allowable delivery range was $\left[T_{i L}, T_{i H}\right]$.

(2) The relationship between the task's satisfaction degree with resource service quality and implementation cost

The implementation cost should first exceed the minimum cost of manufacturing the mould parts borne by manufacturing enterprise; otherwise, the enterprise will make no profit and thus forgo the order. Secondly, if the implementation cost is too high, the customer will go elsewhere and the enterprise will have not have an order to implement. Therefore, the implementation cost must have an acceptable range, denoted as $\left[\mathrm{C}_{\mathrm{L}}, \mathrm{C}_{\mathrm{H}}\right]$, where $\mathrm{C}_{\mathrm{L}}$ denotes the minimum acceptable cost of the manufacturing enterprise and $\mathrm{C}_{\mathrm{H}}$ denotes the maximum implementation cost that the customer will accept.

Within the acceptable implementation cost range, the task's satisfaction degree with resource service quality is in inverse proportion to the implementation cost, suggesting that the task's satisfaction degree with resource service quality decreased gradually with the increase of the implementation cost. Specifically, as the implementation cost rose, the task's satisfaction degree with resource service quality first dropped rapidly; when the implementation cost increased to a certain value, the manufacturing task's satisfaction degree with resource service quality almost disappeared. Therefore, the relationship between the task's satisfaction degree with resource service quality and the implementation cost can be described as a convex function that is convex to the point of origin. Accordingly, this study selected the following exponential function to describe this relationship: 


$$
f_{2}(C)=\left\{\begin{array}{lr}
0 & C>C_{H}, \quad C<C_{L} \\
\frac{1}{e^{\lambda C}} & C_{L}<C<C_{H}
\end{array}\right.
$$

where $\lambda>0$ denotes the user's sensitivity degree to the implementation cost and is also referred to as the cost sensitivity coefficient.

(3) The relationship between the task's satisfaction degree with resource service quality and completion quality

Assuming that some manufacturing errors will occur during the fabrication process, the completion quality of the manufacturing task demanded by the customer shows a certain tolerance, i.e., the highest quality requirement for part fabrication is not necessarily set for the manufacturing task. In this study, the tolerance limit of the completion quality of the manufacturing task was defined as the minimum tolerance of completion quality and denoted as $Q_{L}$, and the part quality was generally characterized by the qualification rate of the processed part in the mould part's actual manufacturing process. Assuming that $Q_{i}$ denotes the qualification rate of a part, the task's satisfaction degree with resource service quality and the part's completion quality satisfies the following function:

$$
f_{3}\left(Q_{i}\right)=\left\{\begin{array}{cc}
0 & Q_{\mathrm{i}}>Q_{i H}, \quad Q_{\mathrm{i}}<Q_{i L} \\
Q_{i} & Q_{i L}<Q_{\mathrm{i}}<Q_{i H}
\end{array}\right.
$$

where $Q_{L}$ and $Q_{H}$ denote the minimum quality tolerance and the maximum qualification rate respectively.

For a lower part qualification rate, the completion quality is lower, and the task's satisfaction degree with resource service quality is lower; alternatively, the higher the part's qualification rate, the higher the satisfaction degree. When the part's qualification rate is lower than the minimum tolerance $Q_{L}$, the satisfaction degree is equal to 0 ; by contrast, the satisfaction degree reaches the maximum, i.e., 1 , when the qualification rate is $100 \%$. Within the range between $Q_{L}$ and $100 \%$, the satisfaction degree increases gradually with the rising qualification rate.

From the above-described relationships between the task's satisfaction degree with resource service quality and delivery date, implementation cost and completion quality, the comprehensive function of the satisfaction degree of the subtask $t_{i k}$ with the service quality of the manufacturing resource node $G_{j}$ can be written as:

$$
\operatorname{CSI}_{i, k, j}=W 1 f_{1}\left(T_{i, k, j}\right)+W 2 f_{2}\left(C_{i, k, j}\right)+W 3 f_{3}\left(Q_{i, k, j}\right)=W 1 f_{1}\left(T_{i, k, j}\right)+W 2 \frac{1}{e^{\lambda C_{i, k, j}}}+W 3 Q_{i, k, j}
$$

Definition 2: The load balance of manufacturing resources

For an optimal allocation of resources including $m \times M$ manufacturing tasks and $n$ manufacturing resource nodes, the load rate of the manufacturing resource, denoted as $\phi_{j}$, can be written as:

$$
\phi_{j}=\frac{\sum_{i=1}^{m} \sum_{k=1}^{u_{j}} x_{i, k, j} \cdot t_{i, k, j}^{e c}}{T p_{j}} \times 100 \%
$$

where $\phi_{j}$ denotes the load rate of the candidate resource node $G_{j}$ (in actual production, $\phi_{j}$ is generally not greater than $80 \%) ; \sum_{i=1}^{m} \sum_{k=1}^{u_{i}} x_{i, k, j} \cdot t_{i, k, j}^{e c}$ denotes the load of the candidate resource node $G_{j}$ and represents the sum of the labor hours of various sub-tasks after the resource nodes were selected for the manufacturing task; $x_{i, k, j}$ denotes the relationship between the $k$-th sub-task in the $i$-th task $t_{i}$ and $n$ candidate resource nodes (if the candidate resource node $G_{j}$ was selected for the $k$-th sub-task in the $i$-th task, the decision factor is equal to 1 ; otherwise, the decision factor is equal to 0 ); $t_{i, k, j}^{e c}$ denotes the predicted processing time of the $k$-th sub-task in the $i$-th task $t_{i}$ at the candidate resource node $G_{j}$; and $T p_{j}$ denotes the rated labor hours of the candidate resource node $G_{j}$. The variance of the resource load rates, denoted as $\phi_{j}$, can be used for 
describing the balance state of the resource load. $S(\phi)$ can be calculated by:

$$
\begin{gathered}
S(\phi)=\frac{1}{n} \sqrt{\sum_{j=1}^{n}\left(\phi_{j}-\phi_{\text {avg }}\right)^{2}} \\
\phi_{\text {avg }}=\frac{1}{n} \sum_{j=1}^{n} \phi_{j}
\end{gathered}
$$

where $\phi_{\text {avg }}$ denotes the overall utilization rate of the candidate resource node (the greater the value of $\phi_{\text {avg }}$, the larger the overall utilization rate). At a smaller $S(\phi)$, the difference between resource nodes was smaller and the load of resource nodes became more balanced.

\subsection{The establishment of the bi-level programming model of the optimal allocation of manufacturing resources under a} manufacturing network environment

According to the problem description, definitions and calculation formulas outlined above, the bi-level programming model of the optimal allocation of manufacturing resources under a manufacturing network environment can be described as:

$$
\begin{aligned}
& (U) \max C S I=\sum_{i=1}^{m}\left(W_{1} \cdot \frac{T_{i H}-\sum_{k=1}^{u_{i}} \sum_{j=1}^{n} x_{i, k, j} \cdot T_{i, k, j}}{T_{i H}-T_{i L}}+W_{2} \cdot \sum_{k=1}^{u_{i}} \sum_{j=1}^{n} x_{i, k, j} \cdot \frac{1}{e^{\lambda C_{i, k, j}}}+W_{3} \cdot \sum_{k=1}^{u_{i}} \sum_{j=1}^{n} \frac{x_{i, k, j} \cdot Q_{i, k . j}}{\sum_{i=1}^{m} u_{i}}\right) \\
& T_{i, k, j}=t_{i, k, j}^{e c}+t_{i, k, j, g}^{\mathrm{lg}} \\
& C_{i, k, j}=c_{i, k, j}^{e c}+c_{i, k, j, g}^{\mathrm{lg}} \\
& \text { s.t. } T_{i \min } \leq \sum_{k=1}^{u_{i}} \sum_{j=1}^{n} T_{i, k, j} x_{i, k, j} \leq T_{i \max } \\
& C_{i \min } \leq \sum_{k=1}^{u_{i}} \sum_{j=1}^{n} C_{i, k, j} x_{i, k, j} \leq C_{i \max } \\
& Q_{i \max }-\sum_{k=1}^{u_{i}} \sum_{j=1}^{n} Q_{i, k, j} x_{i, k, j} \geq 0 \\
& \left\{\begin{array}{c}
x_{i, k, j} \in\{0,1\} \cap \sum_{j=1}^{n} x_{i, k, j}=1 \\
\forall i \in\{1,2, \cdots, m\} \\
\forall k \in\left\{1,2, \cdots, u_{i}\right\}
\end{array}\right.
\end{aligned}
$$

(L) $\max F=S(\phi)$

s.t. $\phi_{j}-80 \% \leq 0$

$$
\left\{\begin{array}{c}
x_{i, k, j} \in\{0,1\} \cap \sum_{j=1}^{n} x_{i, k, j}=1 \\
\forall i \in\{1,2, \cdots, m\} \\
\forall k \in\left\{1,2, \cdots, u_{i}\right\}
\end{array}\right.
$$

where: $t_{i, k, j, g}^{\mathrm{lg}}$ denotes the logistics time between the $k$-th sub-task in the task $t_{i}$ at the candidate resource node $G_{j}$ and the $(\mathrm{k}+1)$-th sub-task in the task $t_{i}$ at the candidate resource node $G_{g} ; c_{i, k, j}^{e c}$ denotes the processing cost of the $k$-th sub-task in the 
task $t_{i}$ at the candidate resource node $G_{j} ; c_{i, k, j, g}^{\mathrm{lg}}$ denotes the logistics cost between the $k$-th sub-task in the task $t_{i}$ at the candidate resource node $G_{j}$ and the $(\mathrm{k}+1)$-th sub-task in the task $t_{i}$ at the candidate resource node $G_{g}$; and $x_{i, k, j}$ denotes the relationship between the $k$-th sub-task in the task $t_{i}$ and $n$ candidate resource nodes. If the candidate resource node $G_{j}$ was selected for the $k$-th sub-task in the task $t_{i}$, the decision factor is equal to 1 ; otherwise, the decision factor is equal to 0 .

Eq. (10) and Eq. (17) represent the objective functions in the upper-level and lower-level programming models; Eqs. (13) (16) represent the upper-level constraints; Eq. (18) and Eq. (19) represent the lower-level constraints. Specifically, Eqs. (13) (15) represent the constraints on delivery date, implementation cost and completion quality, respectively. As shown in Eq. (16) and Eq. (19), a sub-task can only be assigned to a candidate resource node under a manufacturing network environment; Eq. (18) represents the constraint on a resource's load rate.

\section{A solution to the model}

The bi-level programming model for the optimal allocation of manufacturing resources is a typical NP-Hard problem, whose solution space increases rapidly with the increase of the numbers of manufacturing tasks and resources. It is very difficult to obtain the optimal solution to the large-scale optimal allocation of manufacturing resources within a reasonable time period. For this problem, it is more practical to acquire the approximate optimal solution within a reasonable time period.

For the established bi-level programming model of the optimal allocation of manufacturing resources under a manufacturing network environment, this study developed a hierarchical discrete PSO (HDPSO) on the basis of the discrete PSO (DPSO) [9, 10, 20]. A HDPSO integrates two DPSOs for solving both upper-level and lower-lever programming models, and then follows the basic idea of non-dominated sorting in a multi-objective optimization. Finally, it solves the bilevel programming model for the optimal allocation of manufacturing resources through game-based interactive iteration among the populations at different levels.

A PSO is a kind of global adaptive random search technique based on swarm intelligence and is now used mainly for optimal solutions in a continuous domain. There are a number of difficulties in solving complex discrete problems, such as the optimal allocation of resources. To seek the optimal solution in a discrete space, a PSO should be discretized [6]. The DPSO algorithm that aims for optimization in a discrete domain was described in detail in Ref. [12], and is not repeated in this article.

A HDPSO is a DPSO-based optimization algorithm obtained via nested iterations. In each iteration, both the upperlevel and lower-level models were solved using a DPSO. Firstly, the decision variable in the upper-level model, denoted as $\mathrm{X}$, was used in the lower-level model for obtaining the optimal solution $\mathrm{Y}^{*}$; then, the optimal solution $\mathrm{Y}^{*}$ was fed back to the upper-level model, and the upper-level model sought the overall optimal solution $\mathrm{X}^{*}$ within the possible range based on the optimal solution $\mathrm{Y}^{*}$ in the lower-level model. Through interactive iteration between the two levels, both levels in the bi-level programming were optimized simultaneously. Finally, the approximate global optimal solution to the bi-level programming model, denoted as Y, was acquired. Fig. 2 illustrates the procedure of the developed HDPSO algorithm.

The specific procedure for solving a bi-level programming problem using a HDPSO algorithm is now described.

- Step 1: The decision variable, population X, within the range of the decision variables in the upper-level model was initialized.

- Step 2: For each individual in the initial upper-level population, the lower-level population $\mathrm{Y}^{*}$ constituted by the optimal solutions in the lower-level programming model was solved using a DPSO algorithm; then, the lower-lever population $\mathrm{Y}^{*}$ was passed to the upper-level model and used as the basis for the implementation of the algorithm in the upper-level DPSO.

- Step 3: For each individual in the upper-level population, the upper-level programming model was solved using a DPSO, and the upper-level population $\mathrm{X}^{*}$, which was constituted by the optimal solution in the upper-level programming model corresponding to each individual in the lower-level population, was formed.

- Step 4: For the populations in both the upper- and lower-level models, their fitness degrees were evaluated and a Cartesian set was formed. The Cartesian, including two optimization objectives in both levels, should be processed by means of a multi-objective optimization technique. This study employed a Pareto-frontier level measurement operator for sorting the individuals according to the non-dominated relationship in a multi-objective optimization.

- Step 5 The HSPSO ended the assessment process. One of these two indexes, namely, whether the outer loop reached the maximum number of iterations or whether the population's average fitness variation amplitude 
satisfied the preset precision range, was used as the algorithm's end conditions. If one of the above-described conditions was satisfied, the individuals in the frontier set constituted the set of optimal solutions. Finally, an individual in the frontier set was selected according to the actual conditions and adopted as the ultimate solution. If these two conditions were not satisfied, the algorithm returned to Step 2 and repeated.

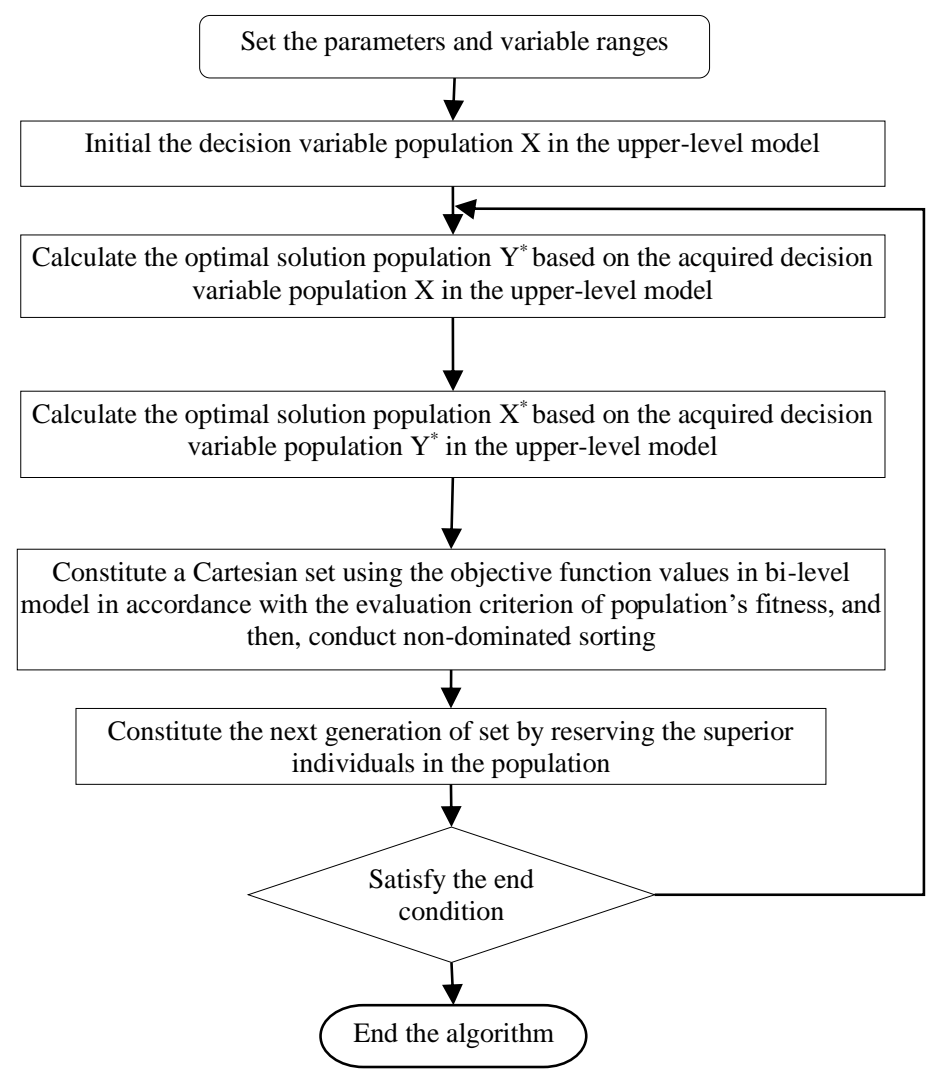

Figure 2. A flowchart of the HDPSO algorithm

\section{An experimental validation and analysis of the results}

\subsection{A case study}

Next, by taking the production order of 20 sets of moulds received by a large-scale mould-manufacturing enterprise as an example, the proposed bi-level programming model for the optimal allocation of mould manufacturing resources and HDPSO algorithm were validated. The manufacturing of each set of moulds included 10 manufacturing tasks, denoted as $t_{1}$, $\mathrm{t}_{2}, \mathrm{t}_{3}, \mathrm{t}_{4}, \mathrm{t}_{5}, \mathrm{t}_{6}, \mathrm{t}_{7}, \mathrm{t}_{8}, \mathrm{t}_{9}$ and $\mathrm{t}_{10}$; then, each manufacturing task was further decomposed into several manufacturing sub-tasks. Each manufacturing sub-task had 10 candidate manufacturing resources, denoted as $\mathrm{G}_{1}, \mathrm{G}_{2}, \mathrm{G}_{3}, \mathrm{G}_{4}, \mathrm{G}_{5}, \mathrm{G}_{6}, \mathrm{G}_{7}, \mathrm{G}_{8}, \mathrm{G}_{9}$ and $\mathrm{G}_{10}$, and the most appropriate manufacturing resource is selected to finish the manufacturing task. Moreover, the manufacturing task should be finished within the allowable delivery date, with an acceptable implementation cost and completion quality; in addition, the overall cost should be as low as possible while the load rate of manufacturing resources should be as balanced as possible. 10 manufacturing network nodes in total provided the required manufacturing resources, and the related information is listed in Tables $1 \sim 3$.

Table 1. Information about the manufacturing task

\begin{tabular}{|c|c|c|c|}
\hline Task & $\begin{array}{l}\text { Delivery date range } \\
\text { ( } T \text {, with a unit of } h \text { ) }\end{array}$ & $\begin{array}{l}\text { Acceptable cost range } \\
\text { (Q, with a unit of yuan) }\end{array}$ & $\begin{array}{l}\text { Minimum qualification rate } \\
\text { (S) }\end{array}$ \\
\hline$t_{1}$ & {$[80,130]$} & {$[400,900]$} & 0.85 \\
\hline$t_{2}$ & {$[70,100]$} & {$[800,1000]$} & 0.92 \\
\hline$t_{3}$ & {$[240,280]$} & {$[1400,2000]$} & 0.85 \\
\hline$t_{4}$ & {$[120,180]$} & {$[700,1000]$} & 0.86 \\
\hline$t_{5}$ & {$[220,280]$} & {$[1000,1400]$} & 0.85 \\
\hline$t_{6}$ & {$[60,90]$} & {$[800,1200]$} & 0.80 \\
\hline
\end{tabular}




\begin{tabular}{|c|c|c|c|}
\hline$t_{7}$ & {$[80,1420]$} & {$[1000,1500]$} & 0.95 \\
\hline$t_{8}$ & {$[170,220]$} & {$[900,1300]$} & 0.80 \\
\hline$t_{9}$ & {$[150,190]$} & {$[800,1200]$} & 0.94 \\
\hline$t_{10}$ & {$[60,100]$} & {$[700,1000]$} & 0.90 \\
\hline
\end{tabular}

\begin{tabular}{|c|c|c|c|c|c|c|c|c|c|c|c|c|c|c|c|c|c|}
\hline Task & Subtask & $\begin{array}{c}\text { Resource } \\
\text { node }\end{array}$ & $\begin{array}{l}\mathrm{T}_{\mathrm{ec}} \\
(\mathrm{h})\end{array}$ & $\begin{array}{c}\mathrm{C}_{\mathrm{ec}} \\
(\mathrm{RMB} \\
\text { yuan })\end{array}$ & Q & Task & Subtask & $\begin{array}{c}\text { Resource } \\
\text { node }\end{array}$ & $\begin{array}{l}\mathrm{T}_{\mathrm{ec}} \\
(\mathrm{h})\end{array}$ & $\begin{array}{c}\mathrm{C}_{\mathrm{ec}} \\
\text { (RMBy } \\
\text { uan) }\end{array}$ & Q & Task & Subtask & $\begin{array}{c}\text { Resource } \\
\text { node }\end{array}$ & $\begin{array}{l}\mathrm{T}_{\mathrm{ec}} \\
(\mathrm{h})\end{array}$ & $\begin{array}{c}\mathrm{C}_{\mathrm{ec}} \\
\text { (RMBy } \\
\text { uan) }\end{array}$ & Q \\
\hline \multirow{9}{*}{$t_{1}$} & \multirow{2}{*}{$t_{1,1}$} & $M G_{2}$ & 18 & 150 & 0.85 & \multirow{9}{*}{$t_{4}$} & \multirow{2}{*}{$t_{4,1}$} & $M G_{9}$ & 45 & 175 & 0.86 & \multirow{2}{*}{\multicolumn{2}{|c|}{$t_{7,1}$}} & $M G_{9}$ & 18 & 375 & 0.96 \\
\hline & & $M G_{4}$ & 22 & 165 & 0.86 & & & $M G_{10}$ & 42 & 165 & 0.88 & & & $M G_{10}$ & 22 & 395 & 0.98 \\
\hline & \multirow{2}{*}{$t_{1,2}$} & $M G_{3}$ & 20 & 125 & 0.86 & & \multirow{2}{*}{$t_{4,2}$} & $M G_{5}$ & 20 & 180 & 0.88 & \multirow{6}{*}{$t_{7}$} & \multirow{2}{*}{$t_{7,2}$} & $M G_{1}$ & 20 & 285 & 0.95 \\
\hline & & $M G_{5}$ & 21 & 135 & 0.88 & & & $M G_{6}$ & 22 & 200 & 0.90 & & & $M G_{3}$ & 22 & 290 & 0.94 \\
\hline & \multirow{3}{*}{$t_{1,3}$} & $M G_{1}$ & 9 & 240 & 0.92 & & \multirow{2}{*}{$t_{4,3}$} & $M G_{7}$ & 37 & 170 & 0.93 & & \multirow[b]{2}{*}{$t_{7,3}$} & $M G_{6}$ & 19 & 255 & 0.95 \\
\hline & & $M G_{2}$ & 12 & 250 & 0.88 & & & $M G_{9}$ & 42 & 210 & 0.96 & & & $M G_{7}$ & 22 & 270 & 0.90 \\
\hline & & $M G_{4}$ & 15 & 235 & 0.90 & & \multirow{3}{*}{$t_{4,4}$} & $M G_{7}$ & 38 & 240 & 0.95 & & \multirow[b]{2}{*}{$t_{7,4}$} & $M G_{5}$ & 22 & 240 & 0.94 \\
\hline & \multirow[b]{2}{*}{$t_{1,4}$} & $M G_{5}$ & 32 & 150 & 0.87 & & & $M G_{8}$ & 42 & 230 & 0.89 & & & $M G_{8}$ & 18 & 230 & 0.96 \\
\hline & & $M G_{6}$ & 32 & 130 & 0.90 & & & $M G_{9}$ & 48 & 210 & 0.92 & \multirow{9}{*}{$t_{8}$} & \multirow[b]{2}{*}{$t_{8,1}$} & $M G_{2}$ & 43 & 210 & 0.82 \\
\hline \multirow{6}{*}{$t_{2}$} & \multirow{2}{*}{$t_{2,1}$} & $M G_{1}$ & 22 & 290 & 0.93 & \multirow{12}{*}{$t_{5}$} & \multirow{2}{*}{$t_{5,1}$} & $M G_{1}$ & 40 & 195 & 0.90 & & & $M G_{4}$ & 37 & 230 & 0.86 \\
\hline & & $M G_{4}$ & 20 & 300 & 0.95 & & & $M G_{2}$ & 36 & 185 & 0.88 & & \multirow[b]{2}{*}{$t_{8,2}$} & $M G_{5}$ & 79 & 170 & 0.84 \\
\hline & \multirow{2}{*}{$t_{2,2}$} & $M G_{6}$ & 24 & 280 & 0.94 & & \multirow{2}{*}{$t_{5,2}$} & $M G_{5}$ & 55 & 170 & 0.85 & & & $M G_{6}$ & 75 & 185 & 0.88 \\
\hline & & $M G_{7}$ & 20 & 265 & 0.95 & & & $M G_{7}$ & 54 & 185 & 0.90 & & & $M G_{6}$ & 28 & 385 & 0.84 \\
\hline & & $M G_{5}$ & 20 & 270 & 0.92 & & \multirow[b]{2}{*}{$t_{5,3}$} & $M G_{2}$ & 19 & 230 & 0.82 & & $t_{8,3}$ & $M G_{8}$ & 30 & 360 & 0.88 \\
\hline & $t_{2,3}$ & $M G_{8}$ & 18 & 265 & 0.94 & & & $M G_{4}$ & 22 & 210 & 0.86 & & & $M G_{3}$ & 28 & 240 & 0.82 \\
\hline & & $M G_{1}$ & 36 & 230 & 0.86 & & & $M G_{1}$ & 36 & 250 & 0.94 & & $t_{8,4}$ & $M G_{6}$ & 25 & 260 & 0.84 \\
\hline & $t_{3,1}$ & $M G_{2}$ & 40 & 240 & 0.88 & & $t_{5,4}$ & $M G_{2}$ & 40 & 280 & 0.89 & & & $M G_{7}$ & 20 & 280 & 0.83 \\
\hline & & $M G_{9}$ & 49 & 220 & 0.90 & & & $M G_{5}$ & 37 & 260 & 0.90 & & & $M G_{1}$ & 43 & 370 & 0.95 \\
\hline & $t_{3,2}$ & $M G_{10}$ & 45 & 195 & 0.92 & & & $M G_{2}$ & 50 & 165 & 0.92 & & $t_{9,1}$ & $M G_{2}$ & 46 & 365 & 0.94 \\
\hline & & $M G_{2}$ & 25 & 180 & 0.85 & & $t_{5,5}$ & $M G_{3}$ & 45 & 180 & 0.88 & & & $M G_{3}$ & 49 & 380 & 0.96 \\
\hline & $t_{3,3}$ & $M G_{3}$ & 20 & 190 & 0.88 & & & $M G_{4}$ & 48 & 170 & 0.94 & & & $M G_{4}$ & 46 & 270 & 0.95 \\
\hline & & $M G_{4}$ & 31 & 190 & 0.85 & & & $M G_{3}$ & 12 & 280 & 0.82 & $t_{9}$ & $t_{9,2}$ & $M G_{5}$ & 47 & 280 & 0.94 \\
\hline$t_{3}$ & & $M G_{7}$ & 56 & 340 & 0.92 & & $t_{6,1}$ & $M G_{5}$ & 13 & 260 & 0.84 & & & $M G_{6}$ & 48 & 295 & 0.94 \\
\hline & $t_{3,4}$ & $M G_{8}$ & 47 & 325 & 0.90 & & & $M G_{4}$ & 23 & 280 & 0.85 & & & $M G_{9}$ & 47 & 260 & 0.96 \\
\hline & & $M G_{8}$ & 44 & 190 & 0.98 & $t_{6}$ & $t_{6,2}$ & $M G_{6}$ & 24 & 265 & 0.92 & & $t_{9,3}$ & $M G_{10}$ & 41 & 275 & 0.94 \\
\hline & $t_{3,5}$ & $M G_{9}$ & 39 & 170 & 0.96 & & & $M G_{2}$ & 32 & 375 & 0.88 & & & $M G_{2}$ & 18 & 375 & 0.92 \\
\hline & & $M G_{2}$ & 50 & 320 & 0.88 & & $t_{6,3}$ & $M G_{3}$ & 29 & 350 & 0.86 & & $t_{10,1}$ & $M G_{4}$ & 20 & 380 & 0.90 \\
\hline & $t_{3,6}$ & $M G_{3}$ & 45 & 300 & 0.86 & & & $M G_{5}$ & 31 & 380 & 0.89 & $t_{10}$ & & $M G_{9}$ & 50 & 350 & 0.92 \\
\hline & & $M G_{4}$ & 55 & 320 & 0.85 & & & & & & & & $t_{10,2}$ & $M G_{10}$ & 55 & 340 & 0.94 \\
\hline
\end{tabular}

Table 3. The relevant data of the candidate resource nodes

\begin{tabular}{|c|c|c|c|c|c|c|c|c|c|c|c|c|c|c|c|c|c|c|c|c|}
\hline \multirow{2}{*}{ resource } & \multicolumn{2}{|c|}{$M G_{1}$} & \multicolumn{2}{|c|}{$M G_{2}$} & \multicolumn{2}{c|}{$M G_{3}$} & \multicolumn{2}{c|}{$M G_{4}$} & \multicolumn{2}{c|}{$M G_{5}$} & \multicolumn{2}{c|}{$M G_{6}$} & \multicolumn{2}{c|}{$M G_{7}$} & \multicolumn{2}{|c|}{$M G_{8}$} & \multicolumn{2}{c|}{$M G_{9}$} & \multicolumn{2}{c|}{$M G_{10}$} \\
\hline & $T_{l g}$ & $C_{l g}$ & $T_{l g}$ & $C_{l g}$ & $T_{l g}$ & $C_{l g}$ & $T_{l g}$ & $C_{l g}$ & $T_{l g}$ & $C_{l g}$ & $T_{l g}$ & $C_{l g}$ & $T_{l g}$ & $C_{l g}$ & $T_{l g}$ & $C_{l g}$ & $T_{l g}$ & $C_{l g}$ & $T_{l g}$ & $C_{l g}$ \\
\hline$M G_{1}$ & & & 5 & 15 & 4 & 20 & 4 & 26 & 5 & 20 & 4 & 30 & 5.0 & 28 & 4 & 20 & 5 & 28 & 5 & 30 \\
\hline$M G_{2}$ & & & & & 3 & 15 & 4 & 25 & 6 & 26 & 5 & 29 & 6 & 38 & 4 & 19 & 4 & 35 & 6 & 40 \\
\hline$M G_{3}$ & & & & & & & 3 & 18 & 3 & 38 & 5 & 28 & 6 & 40 & 2 & 17 & 4 & 28 & 3 & 40 \\
\hline$M G_{4}$ & & & & & & & & 3 & 23 & 4 & 22 & 7 & 30 & 6 & 35 & 5 & 24 & 5 & 30 \\
\hline$M G_{5}$ & & & & & & & & & & 5 & 30 & 4 & 20 & 5 & 30 & 4 & 25 & 6 & 40 \\
\hline$M G_{6}$ & & & & & & & & & & & & 4 & 25 & 7 & 17 & 4 & 35 & 4 & 28 \\
\hline$M G_{7}$ & & & & & & & & & & & & & & & 4 & 32 & 5 & 40 & 4 & 24 \\
\hline$M G_{8}$ & & & & & & & & & & & & & & & & & 4 & 14 & 6 & 40 \\
\hline$M G_{9}$ & & & & & & & & & & & & & & & & & & 5 & 32 \\
\hline$M G_{10}$ & & & & & & & & & & & & & & & & & & & & \\
\hline
\end{tabular}

The establishment of the optimization model and implementation of the algorithm were compiled on MATLABR2010a. The parameters were initialized as follows. The weight coefficients of delivery date, implementation cost and completion quality in the task's satisfaction degree with resource service quality were set as: $\mathrm{w}_{1}=0.3, \mathrm{w}_{2}=0.4$ and $\mathrm{w}_{3}=0.3$ respectively; the number of particles, $m$, was set as 30 ; the length of the particles in a discrete particle group was set as the number of tasks; the particle range was a variable with a range of $0 \sim 1 ; \mathrm{V}_{\text {Max }}$ denotes the maximum velocity and determines the maximum moving distance of a particle in a loop, which is generally set as the particle's range width; the learning factors were set as: $\mathrm{C} 1=\mathrm{C} 2=2$; the maximum number of iterations was set as 200; the rated labor hours at each candidate resource node was set as $4,000 \mathrm{~h}$ and the sensitivity coefficient for the implementation cost was set as $\lambda=0.0001$.

Since the principle of multi-objective non-dominated ranking was used in the design of the proposed HDPSO, the final optimal allocation schemes of resources were not unique and constituted a frontier set of Pareto optimal solutions. Fig. 3 shows the frontier set of the acquired Pareto optimal solutions using the proposed HDPSO algorithm, and Table 4 lists the optimal allocation results of manufacturing resources in the first order, in which the corresponding optimal solutions in the 
upper-level and lower-level models were 54.067 and $0.0375\left(\mathrm{U}^{*}=54.067\right.$ and $\left.\mathrm{L}^{*}=0.0375\right)$ respectively. As shown in Fig. 4 , the average load rate of resource nodes was $61.60 \%$. It can also be observed from Fig. 4 that the maximum utilization rate and the minimum utilization rate of resource nodes were $77.5 \%$ and $38.5 \%$ respectively, suggesting that the load balance among these resource nodes had improved significantly.

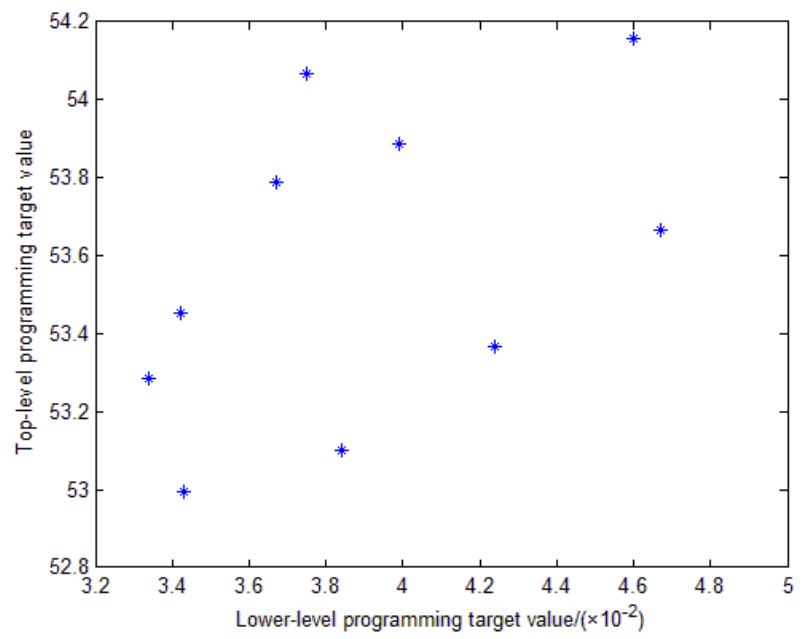

Figure 3. The frontier set of the Pareto optimal solution

Table 4. The optimal resource allocation results in the first order

\begin{tabular}{|c|c|c|c|c|c|c|c|c|}
\hline Task & Subtask & Resource node & Task & Subtask & Resource node & Task & Subtask & Resource node \\
\hline \multirow{4}{*}{$t_{1}$} & $t_{1,1}$ & $M G_{4}$ & \multirow{4}{*}{$t_{4}$} & $t_{4,1}$ & $M G_{10}$ & \multirow{4}{*}{$t_{7}$} & $t_{7,1}$ & $M G_{9}$ \\
\hline & $t_{1,2}$ & $M G_{3}$ & & $t_{4,2}$ & $M G_{6}$ & & $t_{7,2}$ & $M G_{3}$ \\
\hline & $t_{1,3}$ & $M G_{1}$ & & $t_{4,3}$ & $M G_{7}$ & & $t_{7,3}$ & $M G_{6}$ \\
\hline & $t_{1,4}$ & $M G_{5}$ & & $t_{4,4}$ & $M G_{8}$ & & $t_{7,4}$ & $M G_{8}$ \\
\hline \multirow{3}{*}{$t_{2}$} & $t_{2,1}$ & $M G_{1}$ & \multirow{5}{*}{$t_{5}$} & $t_{5,1}$ & $M G_{2}$ & \multirow{4}{*}{$t_{8}$} & $t_{8,1}$ & $M G_{4}$ \\
\hline & $t_{2,2}$ & $M G_{7}$ & & $t_{5,2}$ & $M G_{5}$ & & $t_{8,2}$ & $M G_{6}$ \\
\hline & $\mathrm{t}_{2,3}$ & $M G_{8}$ & & $t_{5,3}$ & $M G_{4}$ & & $t_{8,3}$ & $M G_{8}$ \\
\hline \multirow{6}{*}{$t_{3}$} & $t_{3,1}$ & $M G_{1}$ & & $t_{5,4}$ & $M G_{5}$ & & $t_{8,4}$ & $M G_{7}$ \\
\hline & $t_{3,2}$ & $M G_{10}$ & & $t_{5,5}$ & $M G_{2}$ & \multirow{3}{*}{$t_{9}$} & $t_{9,1}$ & $M G_{1}$ \\
\hline & $t_{3,3}$ & $M G_{3}$ & \multirow{3}{*}{$t_{6}$} & $t_{6,1}$ & $M G_{5}$ & & $t_{9,2}$ & $M G_{4}$ \\
\hline & $t_{3,4}$ & $M G_{8}$ & & $t_{6,2}$ & $M G_{6}$ & & $\mathrm{t}_{9,3}$ & $M G_{9}$ \\
\hline & $t_{3,5}$ & $M G_{9}$ & & $t_{6,3}$ & $M G_{3}$ & \multirow[b]{2}{*}{$t_{10}$} & $t_{10,1}$ & $M G_{2}$ \\
\hline & $t_{3,6}$ & $M G_{3}$ & & & & & $t_{10,2}$ & $M G_{10}$ \\
\hline
\end{tabular}

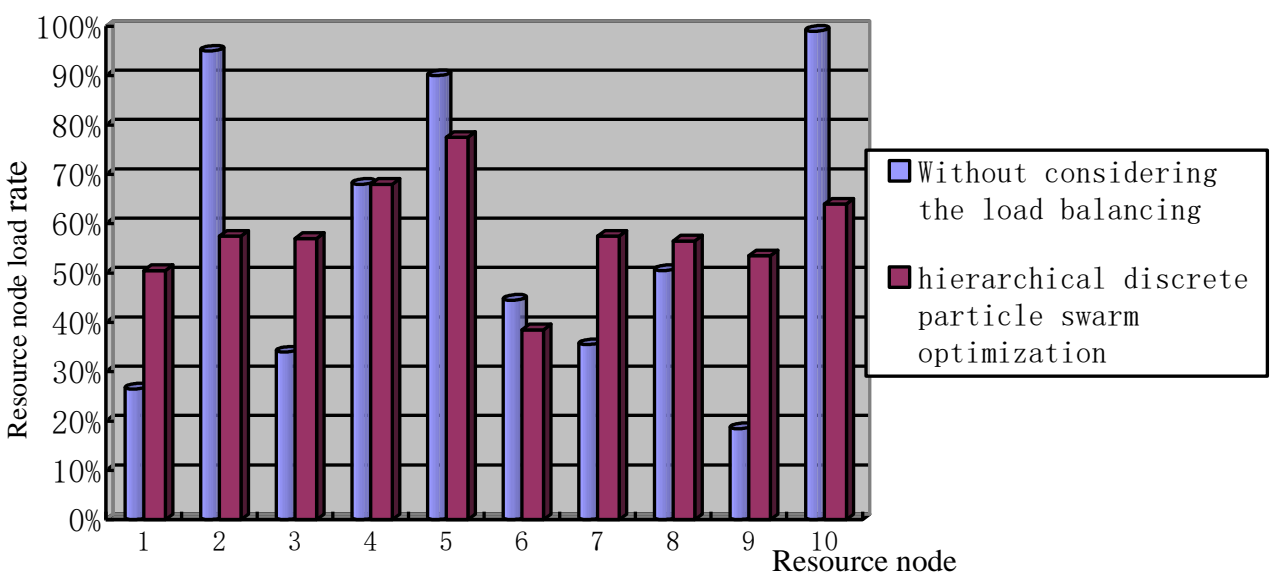

Figure 4. Resource node load rates using two different algorithms

\subsection{A comparison between the results using two different algorithms}

Fig. 4 compares the resource load rates using two different algorithms, namely, the algorithm without the consideration of the balance of resource load and the proposed HDPSO algorithm. Using the algorithm that did not take the balance of resource load into account, the maximum load rate at the resource node was $99.0 \%$ while the minimum load rate was 
$18.5 \%$; by contrast, using the proposed HDPSO, the minimum load rate and the minimum load rate were $77.5 \%$ and $38.5 \%$ respectively, and the distribution was more balanced.

It should be noted that some researchers have adopted other intelligent optimization algorithms to solve bi-level programming problems but generally opted for one optimal solution. Using the proposed algorithm, the frontier set of optimal solutions (also known as the set of optimal solutions) can be acquired, which can provide greater flexibility for the related bi-level programming and decision-making.

\section{Conclusions}

This study first established a bi-level programming model for describing the optimal allocation of mould manufacturing resources, and then employed a HDPSO algorithm for solving this model. Specifically, the upper-level programming model described the satisfaction degree of the manufacturing task with the various service requirements, while the upper-level programming model described the load balance of manufacturing resources. Finally, through a case study, the feasibility of the established model and proposed algorithm was validated. According to the validation results, the algorithm shows a favorable robustness and high calculation efficiency and thus has high practical application values in solving problems relating to the large-scale optimal allocation of manufacturing resources. However, under a manufacturing network environment, the task's satisfaction degree with resource service quality does not just include delivery date, cost and quality. Therefore, in future work, we will focus on the multi-objective optimal allocation model under a manufacturing network environment as well as the related optimization methods.

\section{Acknowledgements}

The work is financially supported by Key University Science Research Project of Jiangsu province of China (13KJA460002), Scientific Foundation of Nanjing Institute of Technology (Grant CKJB201305), and Outstanding Scientific and Technological Innovation Team in Colleges and Universities of Jiangsu Province.

\section{References}

1. J. Z. Fu, Y. L. Zhang, "Study on the optimal disposition method of resources geared to the needs of manufacturing grid," Journal of Machine Design, vol. 23, no. 10, pp. 40-43, 2006

2. J. Z. Fu, Y. L. Zhang, "Resource select ion and optimisation in manufacturing grid based on genetic algorithm," Journal of Chinese Computer Systems, vol. 28, no. 4, pp. 674-677, 2007

3. J. Z. Fu, "A practical resource-searching method for manufacturing grid," The International Journal of Advanced Manufacturing Technology, vol. 74, pp. 335-340, 2014

4. J. Z. Fu, J. D. Liu, "Research on interval transformation of resource in manufacturing grid and multi-objective optimization selection method," Journal of Nanjing University of Information Science and Technology (Natural Science Edition), vol. 5, no. 2, pp. 154-158, 2013

5. J. Z. Fu, "An efficient resource-searching method in manufacturing grid," The International Journal of Advanced Manufacturing Technology, vol. 66, pp. 401-405, 2013

6. W. Han, X. Q. Zhang, "A multisatellite task planning algorithm based on discrete particle swarm," Radio Engineering, vol. 45, no. 1 , pp. 1-5, 2015

7. L. L. Liu, T. Yu, and Z. B. Shi, "Research on QoS -based resource scheduling in manufacturing grid," Computer Integrated Manufacturing Systems, vol. 11, no. 4, pp. 475-480, 2005

8. L. L. Liu, Z. S. Shu, "Resource allocation and network evolution considering economics and robustness in manufacturing grid," The International Journal of Advanced Manufacturing Technology, vol. 57, pp. 393-410, 2011

9. L. J. Li, Z. B. Huang, and F. Liu, "A heuristic particle swarm optimization method for truss structures with discrute variables," Computers and Structures, vol. 87, pp. 435-443, 2009

10. C. B. Li, F. M. Zhang, "Bi-level programming location strategy of logistics distribution center based on hierarchical particle swarm algorithm, "Journal of Lanzhou University of Technology, vol. 39, no. 4, pp. 105-109, 2013

11. X. F. Ma, X. D. Dai, and S. D. Sun, "Optimization deployment of networked manufacturing resources," Computer Integrated Manufacturing Systems, vol. 10, no. 5, pp. 523-527, 2004

12. Q.K. Pan, M. Fatih Tasgetiren, and Y. C. Liang, "A discrete particle swarm optimization algorithm for the no-wait flowshop scheduling problem," Computers and operations research, vol. 35, no. 9, pp. 2807-2839, 2008

13. S. Q. Song, C. M. Ye, "Research on the manufacturing grid resource scheduling problem based on quantum particle swarm algorithm," Manufacturing Automation, vol. 30, no. 10, pp. 40-43, 2008

14. Z. B. Shi, L. L. Liu, "Manufacturing grid and its resource configuration algorithm," Computer Engineering, vol. 30, no. 5, pp. 117-119, 2004

15. F. Tao, L. Zhang, and K. Lu, "Research on manufacturing grid resource service optimal-selection and composition framework," Enterprise Information Systems, vol. 6, no. 2, pp. 237-264, 2012

16. F. Tao, Y. f. Hu, and D. M. Zhao, "Study on resource service match and search in manufacturing grid system," The International Journal of Advanced Manufacturing Technology, vol. 43, pp.379-399,2009 
17. F. Tao, Y. F. Hu, D. M. Zhao, Z. D. Zhou, H. J. Zhang, and Z. Z. Lei, "Study on manufacturing grid resource service QoS modeling and evaluation," The International Journal of Advanced Manufacturing Technology, vol. 41, pp. 1034-1042, 2009

18. C. C. Zhou, G. F. Yin, and Y. C. Wu, "Study on the tactics of manufacturing resource optimal allocation oriented to manufacturing grid," Journal of Sichuan University (Engineering Science Edition) , vol. 41, no. 2, pp. 237-244, 2009

19. X. B. Zhang, Y.Y. Ni, "An interval programming model of manufacture resources optimal allocation in grid," Mathematics in Practice and Theory, vol. 42, no. 6, pp. 18-24, 2012

20. Z. G. Zhao, W. Q. Wang, and S.Y. Huang, "Bi-level programming problem based on improved particle swarm algorithm," Computer Science, vol. 40, no. 11A, pp. 115-119, 2013

Hongyan Hao graduated from the School of Mechanical manufacturing and automation, Southeast University, for the degree of Bachelor, Master and Ph. D. Now she is an associate professor of the School of materials engineering, Nanjing Institute of Technology.

Fanxin Kong graduated from the School of Material processing engineering, Southeast University, for the degree of Bachelor, Master's degree. Now he is a professor of the School of materials engineering, Nanjing Institute of Technology. 